\title{
Hesperetin alleviates renal interstitial fibrosis by inhibiting tubular epithelial-mesenchymal transition in vivo and in vitro
}

\author{
HONG-WEI WANG ${ }^{1 *}$, LEI SHI ${ }^{2 *}$, YAN-PING XU ${ }^{3}$, XING-YA QIN $^{4}$ and QI-ZHI WANG ${ }^{5}$ \\ ${ }^{1}$ Department of Cardiology, People's Hospital of Xianfeng County, Xianfeng, Hubei 445600; Departments of ${ }^{2}$ Oncology \\ and ${ }^{3}$ Scientific Research Office, Renmin Hospital of Wuhan University, Wuhan, Hubei 430060; Departments of \\ ${ }^{4}$ Orthopedics and ${ }^{5}$ Gastroenterology, People's Hospital of Xianfeng County, Xianfeng, Hubei 445600, P.R. China
}

Received November 11, 2016; Accepted June 23, 2017

DOI: $10.3892 /$ etm.2017.4968

\begin{abstract}
Hesperetin (HES) is a flavonoid that has been reported to exert protective effects against cardiac remodeling, lung fibrosis and hepatic fibrosis. However, reports on the effects and potential mechanisms of HES in renal fibrosis are limited. In the present study, a unilateral ureteric obstruction (UUO) mouse model and a transforming growth factor (TGF)- $\beta 1$-activated normal rat kidney (NRK)-52E cell model were established. HES was subsequently administered to these models to evaluate its anti-fibrotic effects and potential underlying mechanisms of action. The results demonstrated that HES reduced obstruction-induced renal injury and deposition of the extracellular matrix components collagen-I and fibronectin in UUO mouse kidneys $(\mathrm{P}<0.05)$. Furthermore, HES treatment significantly suppressed EMT, as evidenced by decreased expression of $\alpha$-smooth muscle actin and E-cadherin, $(\mathrm{P}<0.05)$. Additionally, HES inhibited the hedgehog signaling pathway in UUO mice and TGF- $\beta 1$-treated NRK-52E cells. The present findings indicate that HES treatment may inhibit EMT and renal fibrosis in vivo and in vitro by antagonizing the hedgehog signaling pathway.
\end{abstract}

\section{Introduction}

Chronic kidney disease (CKD) is a prevalent public health problem that will likely affect up to 160 million individuals worldwide by 2020 (1). The majority of CKDs share common pathological features, including glomerular and tubulointerstitial injuries, oxidative stress, chronic inflammation, renal fibrosis and progressive loss in renal function (2).

Correspondence to: Dr Lei Shi, Department of Oncology, Renmin Hospital of Wuhan University, 238 Jiefang Road, Wuhan, Hubei 430060, P.R. China

E-mail: happystudy2015@sina.com

*Contributed equally

Key words: hesperetin, renal fibrosis, epithelial-to-mesenchymal transition, hedgehog pathway
Renal fibrosis is a common pathway that leads to end-stage renal failure regardless of etiology (3). Renal fibrosis is also characterized by the excessive production and deposition of extracellular matrix (ECM) components, and its primary pathogenesis involves glomerular inflammatory cell infiltration, myofibroblast activation and epithelial-mesenchymal transition (EMT) (4). EMT is among the most important causes of renal interstitial fibrosis (5), and is characterized by renal tubular epithelial cells that acquire mesenchymal phenotypes and myofibroblast functions (6). Sonic hedgehog (Shh) is a vertebrate hedgehog ligand that promotes EMT during biliary fibrosis (7). The activation of hedgehog signaling may contribute to renal fibrosis in CKDs (8). Thus, inhibition of hedgehog signaling may suppress EMT and alleviate renal fibrosis.

Hesperetin (HES) is an abundant flavonoid in citrus fruits, and has been indicated to have anti-oxidative, anti-tumorigenic and anti-inflammatory effects (9-11). HES may also exert protective effects against cardiac remodeling (12), lung fibrosis (13) and hepatic fibrosis (14). However, the impacts of HES on renal fibrosis and kidney function are yet to be reported.

On the basis of previous findings, it was hypothesized that HES may ameliorate renal fibrosis by inhibiting hedgehog signaling. To test this hypothesis, the present study investigated the effects of HES in a unilateral ureteral obstruction (UUO) model and in the murine renal proximal tubule cell line, normal rat kidney (NRK)-52E. The effects of HES on renal fibrogenesis and hedgehog signaling were also evaluated.

\section{Materials and methods}

Animals and experimental design. All procedures involving animals were approved by the Ethics Committee for Animal Research of Wuhan University (Wuhan, China). All animals received humane care in compliance with the Guide for the Care and Use of Laboratory Animals prepared by the Institute of Laboratory Animal Resources and the National Research Council (15). A total of 24 male C57BL6 mice (age, 8 weeks; weight, 20-25 g) were purchased from the Animal Center of Wuhan University; these mice were randomly divided into 3 groups $(n=8)$ : Sham operation, UUO + vehicle 
( $0.5 \%$ carboxymethylcellulose, Sigma-Aldrich; Merck KGaA, Darmstadt, Germany) and UUO + $60 \mathrm{mg} / \mathrm{kg} /$ day HES (Sigma-Aldrich; Merck KGaA). The mice were maintained at $25^{\circ} \mathrm{C}$ under a 12 -h light/dark cycle and provided ad libitum access to food and water. UUO was performed in accordance with an established procedure (16). The mice in the UUO + HES group were orally treated with $60 \mathrm{mg} / \mathrm{kg} / \mathrm{day}$ HES from 7 days prior to surgery to 7 days post-surgery. The mice in the UUO + vehicle group were treated with the same volume of vehicle solution ( $0.5 \%$ carboxymethylcellulose). The mice were sacrificed on day 7 post-surgery for renal tissue and blood sampling. Blood samples were harvested from the left renal vein when the mice were sacrificed, and immediately centrifuged at $13,000 \mathrm{xg}$ for $5 \mathrm{~min}$ at $4^{\circ} \mathrm{C}$. Sera were then collected and stored at $-20^{\circ} \mathrm{C}$. Blood urea nitrogen (BUN) and serum creatinine levels were determined using commercially available kits (BUN, 48T/96T, Biofine, Blaine, WA, USA; creatinine, 48T/96T, Shanghai Yanjin Bioscience Company, Shanghai, China) using an AU5800 automatic biochemistry analyzer (Beckman Coulter, Inc., Brea, CA, USA).

Cell culture and experimental design. NRK-52E cells were purchased from Wuhan Boster Bioengineering Co., Ltd. (Wuhan, China) and cultured in Dulbecco's modified Eagle's medium supplemented with $10 \%$ fetal bovine serum (Hyclone; GE Healthcare Life Sciences; Logan, UT, USA) at $37^{\circ} \mathrm{C}$ in a humidified atmosphere containing $5 \% \mathrm{CO}_{2}$. NRK-52E cells were seeded onto a 6 -well dish $\left(3 \times 10^{6}\right.$ cells/well) and starved in serum-free medium for $24 \mathrm{~h}$ at $37^{\circ} \mathrm{C}$, and subsequently treated with $5 \mathrm{ng} / \mathrm{ml}$ recombinant human transforming growth factor (TGF) $-\beta 1$ and 50 or $100 \mu \mathrm{M}$ HES for $24 \mathrm{~h}$ at $37^{\circ} \mathrm{C}$. The cells were subsequently assigned into 4 treatment groups as follows: Control, $5 \mathrm{ng} / \mathrm{ml}$ transforming growth factor (TGF)- $\beta 1$ (positive control; R\&D Systems, Inc., Minneapolis, MN, USA); $5 \mathrm{ng} / \mathrm{ml}$ TGF- $\beta 1+50 \mu \mathrm{M}$ HES; and $5 \mathrm{ng} / \mathrm{ml} \mathrm{TGF}-\beta 1+100 \mu \mathrm{M}$ HES.

Hematoxylin and eosin (H\&E) staining. Renal tissues extracted from C57BL6 mice in each group were placed in $10 \%$ buffered formalin for $24 \mathrm{~h}$ at room temperature, embedded in paraffin, and sliced into $4-\mu \mathrm{m}$ thick sections. The sections were stained with $\mathrm{H} \& \mathrm{E}$ at room temperature for $5 \mathrm{~min}$, and then visualized by light microscopy to assess the extent of renal injury. Interstitial damage was graded as previously described (17).

Immunohistochemistry staining. Immunohistochemical analysis was performed in accordance with an established procedure (18). Kidney tissues were fixed in $4 \%$ paraformaldehyde at room temperature overnight, embedded in paraffin and sliced into $4-\mu \mathrm{m}$ thick sections. Sections were dewaxed and hydrated in a decreasing series of ethanol, and antigens were retrieved in $0.01 \%$ sodium citrate buffer at $100^{\circ} \mathrm{C}$ for $1 \mathrm{~h}$. Endogenous peroxidase activity was reduced using $3 \% \mathrm{H}_{2} \mathrm{O}_{2}$ at room temperature for $10 \mathrm{~min}$ and $5 \%$ bovine serum albumin (Sigma-Aldrich; Merck KGaA) was used to block nonspecific binding at room temperature for up to $1 \mathrm{~h}$. The sections were incubated with the following primary antibodies at $37^{\circ} \mathrm{C}$ for $1 \mathrm{~h}$ : Anti- $\alpha$-smooth muscle actin (SMA, 1:100; BM0002, Wuhan Boster Bioengineering Co., Ltd.), anti-E-cadherin
(1:200; 610181; BD Biosciences, Franklin Lakes, NJ, USA), anti-collagen I (1:100; PB0981; Wuhan Boster Bioengineering Co., Ltd) and anti-fibronectin (FN, 1:200; Ab2413; Abcam, Cambridge, UK). Goat anti-rabbit secondary antibodies labeled with horseradish peroxidase (1:200; BA1080; Wuhan Boster Bioengineering Co., Ltd.) were then added to the tissues and incubated at room temperature for $1 \mathrm{~h}$. Diaminobenzidine (Sigma-Aldrich; Merck KGaA) was added to detect positive signals at room temperature for $10 \mathrm{sec}$. A total of 10 random fields were selected to capture images using an inverted light microscope (Olympus Corporation, Tokyo, Japan). Images were subsequently quantified with Image Pro-Plus 6.0 software (Rockville, MD, USA).

Reverse transcription-quantitative polymerase chain $(R T-q P C R)$. Total RNA was extracted from renal tissues and NRK-52E cells using TRIzol reagent (Invitrogen; Thermo Fisher Scientific, Inc., Waltham, MA, USA) and reverse-transcribed into complementary DNA from 2 ug total RNA using oligo (dT) primers with a Prime Script ${ }^{\mathrm{TM}}$ RT reagent kit (Takara Bio, Inc., Otsu, Japan) according to the manufacturer's instructions. PCR amplifications were conducted with SYBR Premix Ex Taq II (Takara Bio, Inc.) according to the manufacturer's instructions in an AB7500 real-time PCR system (Thermo Fisher Scientific, Inc.) and normalized to GAPDH. The PCR primers used are presented in Table I. The qPCR thermocycling conditions were as follows: $95^{\circ} \mathrm{C}$ for $10 \mathrm{~min}$, followed by by 40 cycles of $5 \mathrm{sec}$ at $95^{\circ} \mathrm{C}, 30 \mathrm{sec}$ at $60^{\circ} \mathrm{C}$ and $1 \mathrm{~min}$ at $72^{\circ} \mathrm{C}$. The $\mathrm{Cq}$ values of each samples were calculated using the $2^{-\Delta \Delta \mathrm{Cq}}$ method (19).

Western blotting. Renal tissues and NRK-52E cells were lysed with radioimmunoprecipitation assay lysis buffer (Biyuntian, Haimen, China) on ice and the lysates were centrifuged for $15 \mathrm{~min}\left(12,000 \mathrm{x} \mathrm{g}, 4^{\circ} \mathrm{C}\right)$. Protein concentrations were determined with a bicinchoninic acid protein assay kit (Sigma-Aldrich; Merck KGaA). Proteins (20 $\mu \mathrm{g}$ ) were separated by $10 \%$ SDS-PAGE and transferred onto polyvinylidene fluoride membranes (EMD Millipore; Billerica, MA, USA). Following blocking with $5 \%$ non-fat milk at room temperature for $2 \mathrm{~h}$, the membranes were probed with the primary antibodies at $4^{\circ} \mathrm{C}$ overnight. The antibodies used were as follows: Anti- $\beta$-actin (1:400; sc-47778; Santa Cruz Biotechnology, Dallas, TX, USA), anti- $\alpha$-SMA (1:1,000; BM0002; Wuhan Boster Bioengineering Co., Ltd.), anti-E-cadherin (1:1,000; 610181; BD Biosciences). Membranes were subsequently incubated with a secondary IRDye ${ }^{\circledR} 800 \mathrm{CW}$-conjugated antibody (1:1,000; 925-32211; LI-COR Biosciences, Lincoln, NE, USA) for $1 \mathrm{~h}$ at room temperature. The signals were visualized with an Odyssey Imaging System (Odyssey; LI-COR Biosciences, USA) and the target protein expression levels were normalized to those of $\beta$-actin. Protein levels were assessed using ImageJ 1.46 software (National Institutes of Health, Bethesda, MD, USA).

Statistical analysis. All experiments were repeated at least three times independently Statistical analyses were performed with SPSS 18.0 (SPSS, Inc., Chicago, IL, USA). Inter-group comparisons were analyzed by one-way analysis of variance followed by Student-Newman-Keuls' method. Data were 
Table I. Reverse transcription-quantitative polymerase chain reaction primers.

\begin{tabular}{lll}
\hline Gene & \multicolumn{1}{c}{ Forward primer sequence, 5'-3' $^{\prime}$} & \multicolumn{1}{c}{ Reverse primers sequence, 5'-3' } \\
\hline Mice & & \\
Collagen-I & AACGGCAAGGTGTTGTGCGATG & AGCTGGGGAGCAAAGTTTCCTC \\
Fibronectin & ACTGGCGTCCCACGATCCGA & GGAGCGGGGGTCCAGGTGAT \\
GAPDH & CAAGGTCATCCATGACAACTTTG & GTCCACCACCCTGTTGCTGTAG \\
$\alpha$-SMA & ACTGGGACGACATGGAAAAG & CAT CTCCAGAGTCCAGCACA \\
E-cadherin & GTCAAACGGCATCTAAAGC & CAAAGACCTCCTGGATAAACT \\
Shh & CGGCAGATATGAAGGGAAGA & TCATCACAGATGGCCAAGG \\
Gli 1 & CCTGGTGGCTTTCATCAACT & ACACAGGGCTGGACTCCATA \\
NRK-52E cells & & \\
$\alpha-S M A$ & GACCCTGAAGTATCCGATAGA & CACGCGAAGCTCGTTATAGAAG \\
E-cadherin & ACAAACGAGGGCATTCTGAAAACA & CACTGTCACGTGCAGAATGTACTG \\
Shh & ACAAGAAACTCCGAACGATT & ACAAGAAACTCCGAACGATT \\
Gli 1 & CCTCGTGGCTTTCATCAACTCT & GAAGCATCATTGAACCCTGAGTAGA \\
GAPDH & GATGCTGGTGCTGAGTATGRCG & GTGGTGCAGGATGCATTGCTCTGA \\
\hline
\end{tabular}

SMA, smooth muscle actin; Shh, Sonic hedgehog; normal rat kidney-52E.

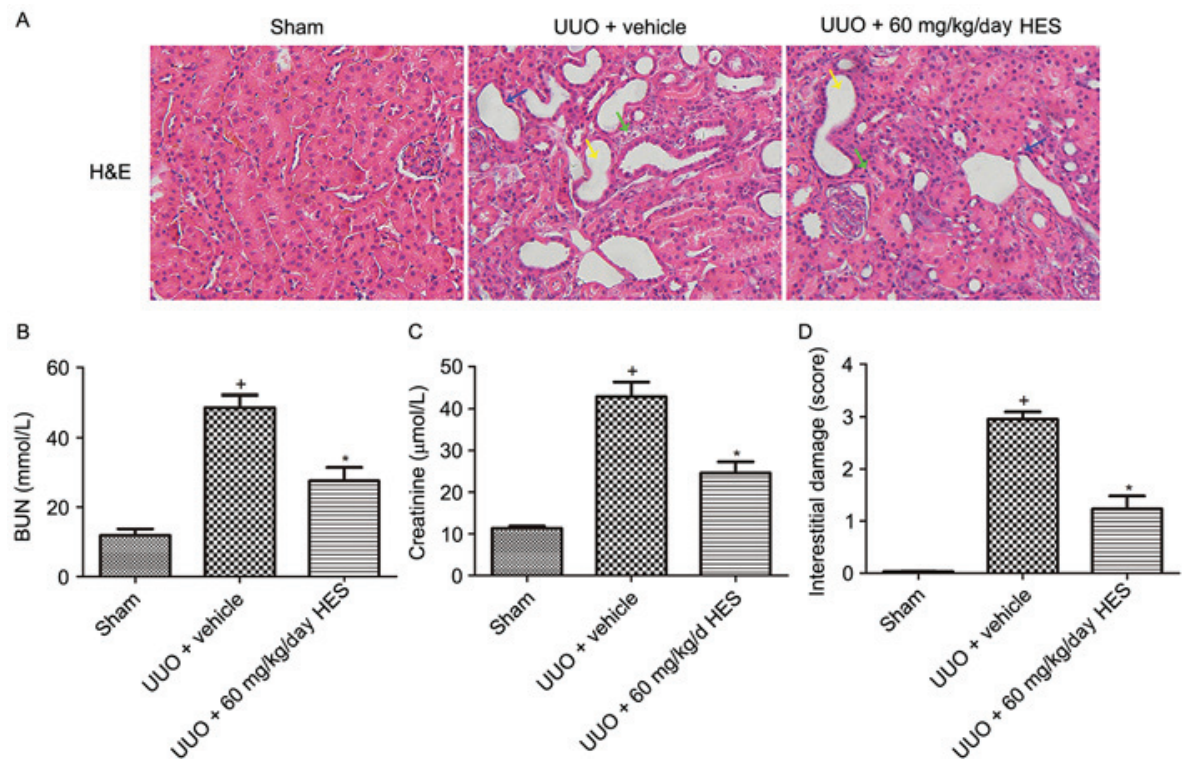

Figure 1. HES inhibited renal damage and decreased the levels of BUN and creatinine in the blood of UUO mice. (A) H\&E staining of the renal tissues isolated from the UUO mice groups (Sham, UUO + vehicle and UUO + $60 \mathrm{mg} / \mathrm{kg} /$ day HES; magnification, $\mathrm{x} 200$ ). (B and C) Blood levels of BUN and creatinine in the 3 groups. (D) Interstitial damage score. Data are presented as the mean \pm standard deviation. Green arrow indicates inflammatory cell infiltration; blue arrow indicates epithelial cell necrosis; yellow arrow indicates tubular dilation. ${ }^{+} \mathrm{P}<0.05$ vs. Sham group, ${ }^{*} \mathrm{P}<0.05$ vs. UUO + vehicle group. H\&E, hematoxylin and eosin; UUO, unilateral ureteric obstruction; HES, hesperetin; BUN, blood urea nitrogen.

expressed as the mean + standard deviation. All P-values were two-sided and $\mathrm{P}<0.05$ was considered to indicate a statistically significant difference.

\section{Results}

Effect of HES on renal dysfunction in UUO mice. Renal histology and BUN and creatinine levels in the blood were assessed to determine the effect of HES on renal function. H\&E staining identified epithelial cell necrosis, inflammatory cell infiltration and tubular dilation in the renal interstitium of the UUO kidneys (Fig. 1A). Furthermore, results suggested that the levels of BUN and creatinine in the UUO + vehicle group were significantly elevated when compared with those in the sham group $(\mathrm{P}<0.05$; Fig. $1 \mathrm{~B}$ and $\mathrm{C})$. Following treatment with HES (60 mg/kg/day), the BUN and creatinine levels significantly declined in UUO mice $(\mathrm{P}<0.05$; Fig. $1 \mathrm{~B}$ and $\mathrm{C}$, respectively). Additionally, the significant damage in the renal interstitium induced by UUO $(\mathrm{P}<0.05)$ was significantly alleviated by treatment with HES ( $\mathrm{P}<0.05$; Fig. $1 \mathrm{~A}$ and $\mathrm{D})$. Therefore, HES may prevent renal dysfunction induced by ureteral obstruction. 

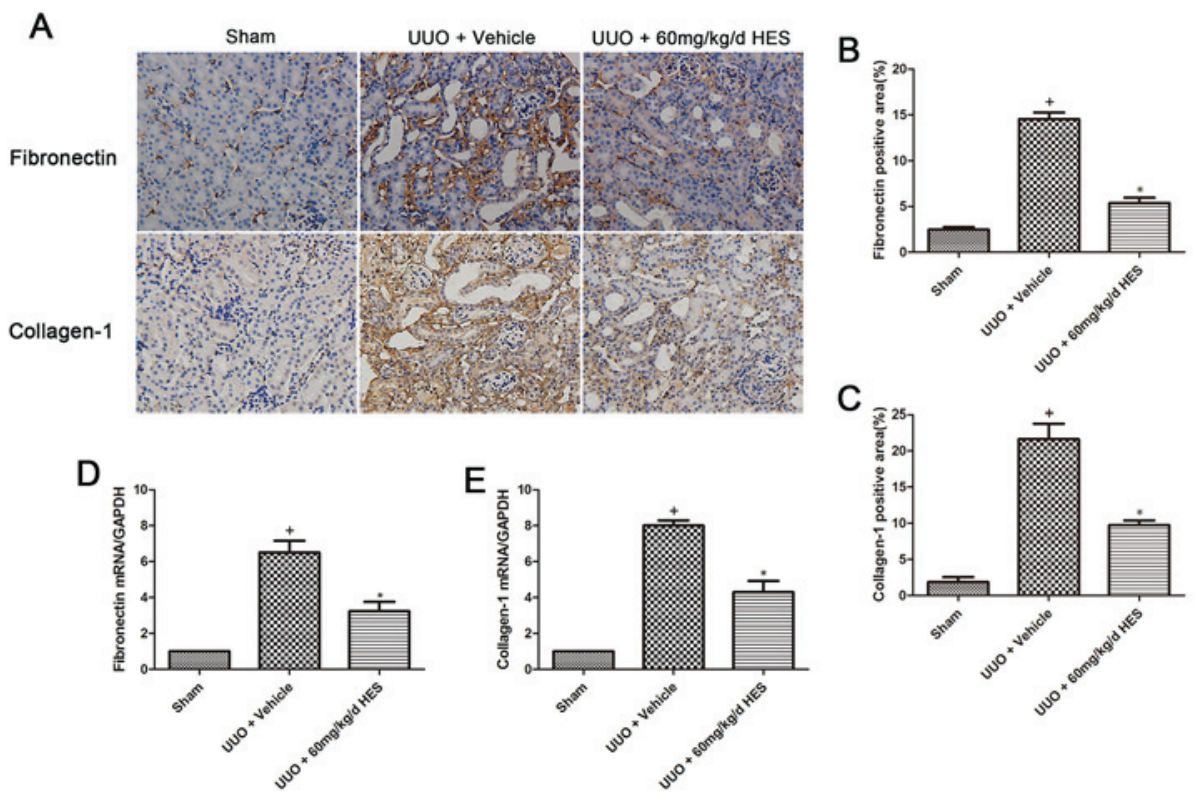

Figure 2. HES reduced the accumulation of extra cellular matrix components in UUO mice. (A) Immunohistochemistry staining of kidney sections isolated from the UUO mice groups with antibodies targeting FN and collagen I. Positive signals were detected with diaminobenzidine (brown; magnification, x200). Semi-quantitative analysis of immunohistochemistry staining for (B) FN and (C) collagen I. mRNA expression levels of (D) FN and (E) collagen I. Data are presented as the mean \pm standard deviation. ${ }^{+} \mathrm{P}<0.05$ vs. Sham group, ${ }^{\prime} \mathrm{P}<0.05$ vs. UUO + Vehicle group. UUO, unilateral ureteric obstruction; HES, hesperetin; FN, fibronectin.
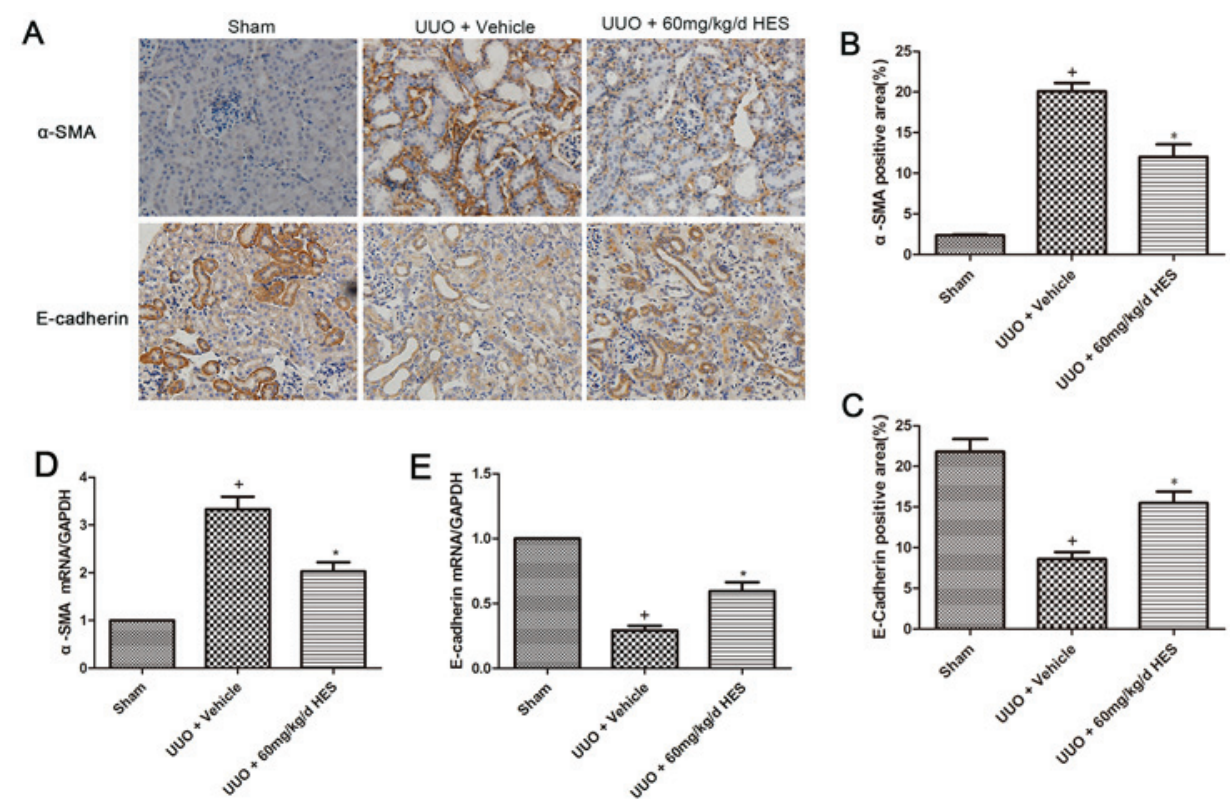

Figure 3. HES blocked EMT in the UUO mice model. (A) Immunohistochemistry staining of kidney sections isolated from the UUO mice groups with antibodies targeting $\alpha$-SMA and E-cadherin. Positive signals were detected with diaminobenzidine (brown; magnification, x200). Semi-quantitative analysis of immunohistochemistry staining for (B) $\alpha$-SMA and (C) E-cadherin. mRNA expression levels of (D) $\alpha$-SMA and (E) E-cadherin in each group. Data were presented as the mean \pm standard deviation. ${ }^{+} \mathrm{P}<0.05$ vs. Sham group, ${ }^{*} \mathrm{P}<0.05$ vs. UUO + Vehicle group. SMA, smooth muscle actin; UUO, unilateral ureteric obstruction; HES, hesperetin.

Effect of HES on ECM deposition and EMT in UUO mice. UUO mice are commonly used animal models of renal interstitial fibrosis (20). The primary feature of renal interstitial fibrosis is the accumulation of ECM components, including FN and collagen (21). As depicted in Fig. 2A, immunohistochemistry staining indicated that HES treatment significantly reversed the accumulation of $\mathrm{FN}$ and collagen I protein in UUO mice $(\mathrm{P}<0.05$; Fig. $2 \mathrm{~B}$ and $\mathrm{C})$. Similarly, RT-qPCR analysis demonstrated that the elevated mRNA expression levels of FN and collagen I in UUO mice $(\mathrm{P}<0.05)$ were significantly reduced by HES treatment $(\mathrm{P}<0.05$; Fig. $2 \mathrm{D}$ and E). Myofibroblasts are considered to be a primary renal interstitial cell responsible for the production of ECM during fibrosis (22). Furthermore, EMT activation has been associated with myofibroblast production (23). In the present study, the protein expression levels of $\alpha$-SMA were upregulated in 


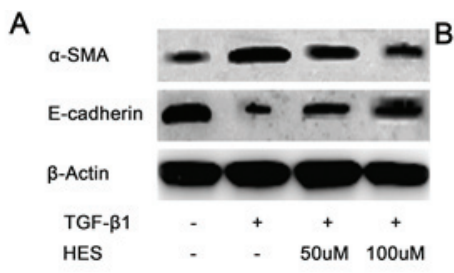

D

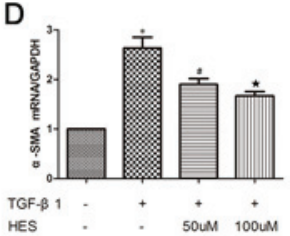

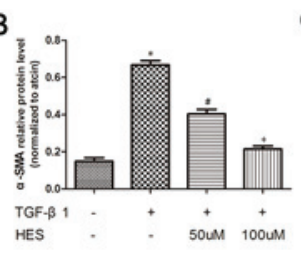

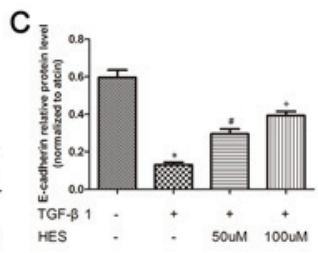

E

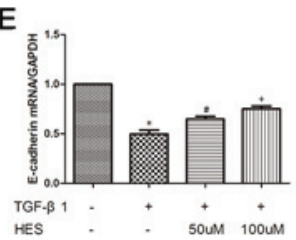

Figure 4. HES suppressed EMT in NRK-52E cells in vitro. (A-C) Western blotting was performed to determine the protein expression levels of $\alpha$-SMA and E-cadherin in the NRK-52E cell groups. (D and E) mRNA expression levels of (D) $\alpha$-SMA and (E) E-cadherin in the 4 cell groups. ${ }^{*} \mathrm{P}<0.05$ vs. control group (untreated cells), ${ }^{\prime} \mathrm{P}<0.05$ vs. TGF- $\beta 1$ group, ${ }^{+} \mathrm{P}<0.05$ vs. $5 \mathrm{ng} / \mathrm{ml}$ TGF- $\beta 1+50 \mu \mathrm{M}$ HES group and ${ }^{*} \mathrm{P}>0.05 \mathrm{vs} .5 \mathrm{ng} / \mathrm{ml}$ TGF- $\beta 1+50 \mu \mathrm{M}$ HES group. Data are presented as the mean \pm standard deviation. SMA, smooth muscle actin; UUO, unilateral ureteric obstruction; HES, hesperetin; TGF, transforming growth factor; NRK-52E, normal rat kidney-52E.

A

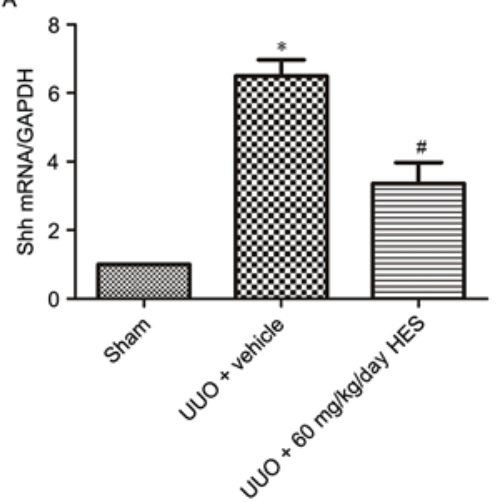

C

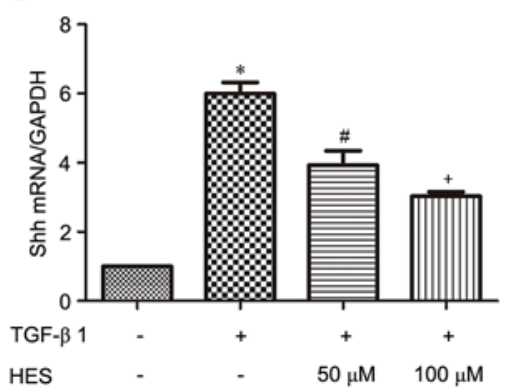

B

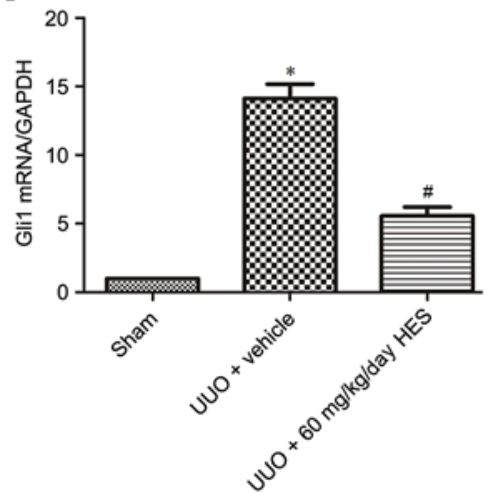

D

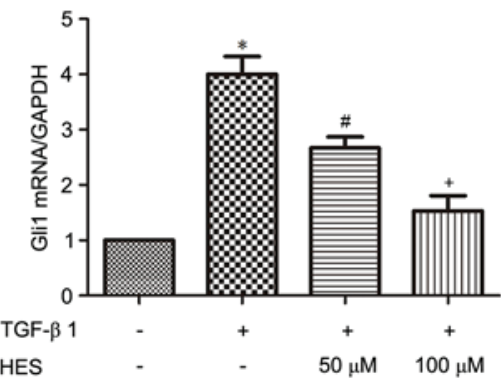

Figure 5. HES suppressed hedgehog signaling in UUO mice and TGF- $\beta 1$-treated NRK-52E cells. (A-D) mRNA expression levels of Shh and Gil in (A and B) UUO mice and (C and D) TGF- $\beta 1$-treated NRK-52E cells with or without HES. Data are presented as the mean \pm standard deviation. For A and B, ${ }^{*} \mathrm{P}<0.05$ vs. sham group, and ${ }^{~} \mathrm{P}<0.05$ vs. $\mathrm{UUO}+$ vehicle group. For $\mathrm{C}$ and $\mathrm{D},{ }^{*} \mathrm{P}<0.05$ vs. control group (untreated cells), ${ }^{\text {"P }} \mathrm{P}<0.05$ vs. TGF- $\beta 1$ group and ${ }^{+} \mathrm{P}<0.05$ vs. $5 \mathrm{ng} / \mathrm{ml}$ TGF- $\beta 1+50 \mu \mathrm{M}$ HES group. Shh, Sonic hedgehog; UUO, unilateral ureteric obstruction; HES, hesperetin; TGF, transforming growth factor; NRK-52E, normal rat kidney-52E.

UUO mice, while those of E-cadherin were downregulated when compared with the sham group; however, these changes were significantly reversed following treatment with HES (Fig. 3A-C). Similarly, the mRNA expression levels of $\alpha$-SMA were significantly upregulated and those of E-cadherin were significantly downregulated in UUO mice $(\mathrm{P}<0.05)$, and these alterations were significantly reversed by HES treatment $(\mathrm{P}<0.05$; Fig. 3D and $\mathrm{E})$.

Effect of HES on EMT in TGF- $\beta 1$-treated NRK-52E cells. Although the in vivo studies indicated that HES may inhibit
EMT, in vitro experiments were performed to further investigate the effects of HES. Western blot analysis demonstrated that 24 -h treatment with TGF- $\beta 1$ altered the protein expression levels of $\alpha$-SMA and E-cadherin in NRK-52E cells (Fig. 4A). Quantification of these results (Fig. 4B and C) indicated that TGF- $\beta 1$ significantly increased the protein expression levels of $\alpha$-SMA and significantly decreased those of E-cadherin in NRK-52E cells when compared with the control group $(\mathrm{P}<0.05)$. These changes were significantly reversed by HES in a dose-dependent manner $(\mathrm{P}<0.05$; Fig. $4 \mathrm{~B}$ and $\mathrm{C})$. Thus, a greater dose of HES $(100 \mu \mathrm{M})$ elicited a stronger inhibitory 
effect on EMT. These results were consistent with the RT-qPCR data (Fig. 4D and E); however, the mRNA expression levels of $\alpha$-SMA between the $5 \mathrm{ng} / \mathrm{ml}$ TGF- $\beta 1+50 \mu \mathrm{M}$ HES and $5 \mathrm{ng} / \mathrm{ml}$ TGF- $\beta 1+100 \mu \mathrm{M}$ HES groups did not differ significantly. Though notably, TGF- $\beta 1$ significantly increased the mRNA expression levels of $\alpha$-SMA and significantly decreased those of E-cadherin in NRK-52E cells $(\mathrm{P}<0.05)$, and these changes were significantly reversed following HES intervention $(\mathrm{P}<0.05$; Fig. 4D and $\mathrm{E})$.

Effect of HES on hedgehog signaling in UUO mouse kidneys and TGF- $\beta 1$-treated NRK-52E cells. In vivo experimental results indicated that the mRNA expression levels of Shh and Gli1 were significantly increased in the UUO group when compared with the sham group $(\mathrm{P}<0.05)$. These increased levels were significantly reduced by HES treatment $(\mathrm{P}<0.05$; Fig. 5A and $\mathrm{B}$, respectively), thus suggesting that HES inhibited hedgehog signaling in UUO mice.

Consistent with these in vivo data, the results of in vitro experiments indicated that the mRNA expression levels of Shh and Gli1 were upregulated by $24-\mathrm{h}$ treatment with TGF- $\beta 1$ in NRK-52E cells, and this effect was significantly reversed by HES treatment $(\mathrm{P}<0.05$; Fig. $5 \mathrm{C}$ and $\mathrm{D})$. Additionally, the higher dose of HES $(100 \mu \mathrm{M})$ inhibited hedgehog signaling to a greater extent.

\section{Discussion}

UUO is commonly used to establish models of renal interstitial fibrosis and renal failure. In the present study, HES exerted protective effects by reducing kidney injury and maintaining kidney functions in UUO mice. HES also prevented the deposition of ECM components associated with EMT. Furthermore, results indicated that the underlying mechanisms regarding the inhibitory effects of HES on EMT may have been associated with inactivation of the hedgehog signaling pathway.

Renal interstitial fibrosis is a major pathological change that occurs in progressive kidney diseases, and is characterized by excessive deposition of ECM components in the cortical interstitium (24). FN and collagen are the primary components of the ECM (21). In UUO mice, HES significantly reduced the upregulated expression levels of FN and collagen, thus indicating a downregulatory effect of HES on ECM production.

Myofibroblasts are terminally differentiated cells responsible for the synthesis of interstitial ECM components (25). However, the source of myofibroblasts has remained controversial. During EMT, epithelial cells are converted into myofibroblasts, which is characterized by the loss of epithelial cell adhesion molecules and the acquisition of mesenchymal cell characteristics, including $\alpha$-SMA and fibroblast surface protein expression $(25,26)$. In kidney fibrosis, EMT is considered to be among the major mechanisms of interstitial myofibroblast generation (27). However, Le Bleu et al (28) observed that the majority of interstitial myofibroblasts were derived from the proliferation of resident interstitial fibroblast, and only $15 \%$ of myofibrolasts were derived from EMT-mediated differentiation. The role of EMT in the generation of interstitial myofibroblasts has further been questioned, due to a lack of data supporting the suggestion that epithelial cells may become activated and cross the tubular basement membrane (29). Furthermore, results have indicated that a partial EMT program may stimulate the differentiation of renal epithelial cells and promote kidney fibrosis (30). In the present study, EMT was activated and accompanied by excessive deposition of ECM components in UUO kidneys. These findings suggest that EMT may be involved in renal fibrogenesis. After NRK-52E cells were treated with HES, the apparent EMT activation was significantly inhibited, as indicated by a dose-dependent decrease in $\alpha$-SMA expression and concomitant increase in E-cadherin expression. These findings were supported by in vitro results, which indicated that HES inhibited TGF- $\beta 1$-induced EMT in NRK-52E cells.

The present study indicates that HES may partially prevent renal fibrosis by inhibiting EMT activation. However, the underlying mechanism remains unknown. A number of crucial signaling molecules, including proteins in the hedgehog family (31), WNT family (32), interleukin family (33) and TGF- $\beta$ super family (34), may induce or contribute to EMT. In hepatic fibrosis, the hedgehog signaling pathway is activated by the binding of its ligands, including Shh, to its membrane receptor patched 1 (Ptch1). The binding of ligand to Ptch1 relieves the signal transducer Smoothened and subsequently activates a cascade; this phenomenon leads to the translocation of Gli1 to the nucleus (35). Fabian et al (36) documented that hedgehog signaling may have an important role in kidney fibrosis and renal development in a paracrine manner. Hedgehog signaling may also promote myofibroblast activation and EMT by directly controlling the expression of a series of fibrogenic genes, including Gli1, Snaill, desmin, FN, $\alpha$-SMA and type I collagen (37). In the present study, the effects of HES on hedgehog signaling activation were investigated. The results indicated that hedgehog signaling was activated in TGF- $\beta 1$-treated NRK-52E cells and UUO mouse kidneys, and the associated molecules were upregulated. The overactivity of hedgehog signaling was downregulated following HES treatment. Thus, the present study provided a potential molecular basis for the inhibition of EMT by HES, involving blockade of the hedgehog signaling pathway.

In conclusion, the present findings suggest that HES may be a potential therapeutic agent for the prevention of progressive renal tubulointerstitial fibrosis and inhibition of EMT. The possible molecular mechanism may involve HES-induced inhibition of hedgehog signaling activation. Therefore, HES may be a therapeutic agent for the treatment of renal fibrosis.

\section{Acknowledgements}

The present study was supported by the Science Research Project of the Health and Family Planning Commission of Hubei Province (grant no. WJ2015Z028), and the Agricultural and Social Science Development Plan of Enshi State in Hubei Province.

\section{References}

1. Jha V, Garcia-Garcia G, Iseki K, Li Z, Naicker S, Plattner B, Saran R, Wang AY and Yang CW: Chronic kidney disease: Global dimension and perspectives. Lancet 382: 260-272, 2013. 
2. Wang Y, Wang B, Du F, Su X, Sun G, Zhou G, Bian X and Liu N: Epigallocatechin-3-gallate attenuates oxidative stress and inflammation in obstructive nephropathy via NF- $\mathrm{BB}$ and Nrf2/HO-1 signalling pathway regulation. Basic Clin Pharmacol Toxicol 117: 164-172, 2015.

3. Liu J, Wei Q, Guo C, Dong G, Liu Y, Tang C and Dong Z: Hypoxia, HIF, and associated signaling networks in chronic kidney disease. Int J Mol Sci 18: pii: E0950, 2017.

4. Yamaguchi Y, Iwano M, Suzuki D, Nakatani K, Kimura K, Harada K, Kubo A, Akai Y, Toyoda M, Kanauchi M, et al: Epithelial-mesenchymal transition as a potential explanation for podocyte depletion in diabetic nephropathy. Am J Kidney Dis 54: 653-664, 2009.

5. Liu Y: New insights into epithelial-mesenchymal transition in kidney fibrosis. J Am Soc Nephrol 21: 212-222, 2010.

6. Zeisberg EM, Potenta SE, Sugimoto H, Zeisberg M and Kalluri R Fibroblasts in kidney fibrosis emerge via endothelial-to-mesenchymal transition. J Am Soc Nephrol 19: 2282-2287, 2008.

7. Omenetti A, Bass LM, Anders RA, Clemente MG, Francis H, Guy CD, McCall S, Choi SS, Alpini G, Schwarz KB, et al: Hedgehog activity, epithelial-mesenchymal transitions, and biliary dysmorphogenesis in biliary atresia. Hepatology 53: 1246-1258, 2011

8. Ding H, Zhou D, Hao S, Zhou L, He W, Nie J, Hou FF and Liu Y: Sonic hedgehog signaling mediates epithelial-mesenchymal communication and promotes renal fibrosis. J Am Soc Nephrol 23: 801-813, 2012.

9. Kim JY, Jung KJ, Choi JS and Chung HY: Modulation of the age-related nuclear factor-kappaB (NF-kappaB) pathway by hesperetin. Aging Cell 5: 401-411, 2006.

10. Ye L, Chan FL, Chen S and Leung LK: The citrus flavonone hesperetin inhibits growth of aromatase-expressing MCF-7 tumor in ovariectomized athymic mice. J Nutr Biochem 23: $1230-1237,2012$

11. Morin B, Nichols LA, Zalasky KM, Davis JW, Manthey JA and Holland LJ: The citrus flavonoids hesperetin and nobiletin differentially regulate low density lipoprotein receptor gene transcription in HepG2 liver cells. J Nutr 138: 1274-1281, 2008.

12. Deng W, Jiang D, Fang Y, Zhou H, Cheng Z, Lin Y, Zhang R, Zhang J, Pu P, Liu Y, et al: Hesperetin protects against cardiac remodelling induced by pressure overload in mice. J Mol Histol 44: 575-585, 2013.

13. Seyedrezazadeh E, Kolahian S, Shahbazfar AA, Ansarin K, Pour Moghaddam M, Sakhinia M, Sakhinia E and Vafa M: Effects of the flavanone combination hesperetin-naringenin, and orange and grapefruit juices, on airway inflammation and remodeling in a murine asthma model. Phytother Res 29: 591-598, 2015.

14. Lin X, Kong LN, Huang C, Ma TT, Meng XM, He Y, Wang QQ and Li J: Hesperetin derivative-7 inhibits PDGF-BB-induced hepatic stellate cell activation and proliferation by targeting Wnt/3-catenin pathway. Int Immunopharmacol 25: 311-320, 2015.

15. Norton JN, Reynolds RP, Chan C, Valdivia RH and Staats HF Assessing the satisfaction and burden within an academic animal care and use program. FASEB J, May 17, 2017 (Epub ahead of print).

16. Shan G, Zhou XJ, Xia Y and Qian HJ: Astragalus membranaceus ameliorates renal interstitial fibrosis by inhibiting tubular epithelial-mesenchymal transition in vivo and in vitro. Exp Ther Med 11: 1611-1616, 2016.

17. Lin SL, Chen RH, Chen YM, Chiang WC, Lai CF, Wu KD and Tsai TJ: Pentoxifylline attenuates tubulointerstitial fibrosis by blocking Smad3/4-activated transcription and profibrogenic effects of connective tissue growth factor. J Am Soc Nephrol 16 $2702-2713,2005$

18. Xu C, Wang W, Xu M and Zhang J: Asiatic acid ameliorates tubulointerstitial fibrosis in mice with ureteral obstruction. Exp Ther Med 6: 731-736, 2013.
19. Livak KJ and Schmittgen TD: Analysis of relative gene expression data using real-time quantitative PCR and the 2(-Delta Delta C(T)) method. Methods 25: 402-408, 2001.

20. Chevalier RL, Forbes MS and Thornhill BA: Ureteral obstruction as a model of renal interstitial fibrosis and obstructive nephropathy. Kidney Int 75: 1145-1152, 2009.

21. Liu Y: Renal fibrosis: New insights into the pathogenesis and therapeutics. Kidney Int 69: 213-217, 2006

22. Sun YB, Qu X, Caruana G and Li J: The origin of renal fibroblasts/myofibroblasts and the signals that trigger fibrosis. Differentiation 92: 102-107, 2016.

23. Lovisa S, Zeisberg M and Kalluri R: Partial epithelial-to-mesenchymal transition and other new mechanisms of kidney fibrosis. Trends Endocrinol Metab 27: 681-695, 2016.

24. Kalluri R and Weinberg RA: The basics of epithelial-mesenchymal transition. J Clin Invest 119: 1420-1428, 2009.

25. Meran S and Steadman R: Fibroblasts and myofibroblasts in renal fibrosis. Int J Exp Pathol 92: 158-167, 2011.

26. Zeisberg M and Duffield JS: Resolved: EMT produces fibroblasts in the kidney. J Am Soc Nephrol 21: 1247-1253, 2010.

27. Strutz F, Okada H, Lo CW, Danoff T, Carone RL, Tomaszewski JE and Neilson EG: Identification and characterization of a fibroblast marker: FSP1. J Cell Biol 130: 393-405, 1995.

28. LeBleu VS, Teng Y, O'Connell JT, Charytan D, Müller GA, Müller CA, Sugimoto $H$ and Kalluri R. Identification of human epididymis protein-4 as a fibroblast-derived mediator of fibrosis. Nat Med 19: 227-231, 2013.

29. Humphreys BD, Lin SL, Kobayashi A, Hudson TE, Nowlin BT, Bonventre JV, Valerius MT, McMahon AP and Duffield JS: Fate tracing reveals the pericyte and not epithelial origin of myofibroblasts in kidney fibrosis. Am J Pathol 176: 85-97, 2010.

30. Grande MT, Sánchez-Laorden B, López-Blau C, De Frutos CA, Boutet A, Arévalo M, Rowe RG, Weiss SJ, López-Novoa JM and Nieto MA: Snaill-induced partial epithelial-to-mesenchymal transition drives renal fibrosis in mice and can be targeted to reverse established disease. Nat Med 21: 989-997, 2015.

31. Lu H, Chen B, Hong W, Liang Y and Bai Y: Transforming growth factor- $\beta 1$ stimulates hedgehog signaling to promote epithelial-mesenchymal transition after kidney injury. FEBS J 283: 3771-3790, 2016.

32. Holland JD, Klaus A, Garratt AN and Birchmeier W: Wnt signaling in stem and cancer stem cells. Curr Opin Cell Biol 25: 254-264, 2013

33. Li Y, Yang J, Dai C, Wu C and Liu Y: Role for integrin-linked kinase in mediating tubular epithelial to mesenchymal transition and renal interstitial fibrogenesis. J Clin Invest 112: 503-516, 2003.

34. Yang J and Liu Y: Dissection of key events in tubular epithelial to myofibroblast transition and its implications in renal interstitial fibrosis. Am J Pathol 159: 1465-1475, 2001.

35. Syn WK, Jung Y, Omenetti A, Abdelmalek M, Guy CD, Yang L, Wang J, Witek RP, Fearing CM, Pereira TA, et al: Hedgehog-mediated epithelial-to-mesenchymal transition and fibrogenic repair in nonalcoholic fatty liver disease. Gastroenterology 137: 1478-1488.e8, 2009.

36. Fabian SL, Penchev RR, St-Jacques B, Rao AN, Sipila P, West KA, McMahon AP and Humphreys BD: Hedgehog-Gli pathway activation during kidney fibrosis. Am J Pathol 180: 1441-1453, 2012.

37. Rowe RG, Li XY, Hu Y, Saunders TL, Virtanen I, Garcia de Herreros A, Becker KF, Ingvarsen S, Engelholm LH, Bommer GT, et al: Mesenchymal cells reactivate Snaill expression to drive three-dimensional invasion programs. J Cell Biol 184: 399-408, 2009. 